\title{
Tout est dit
}

\section{Anna Sax}

Lic. oec. publ., MHA, membre de la rédaction

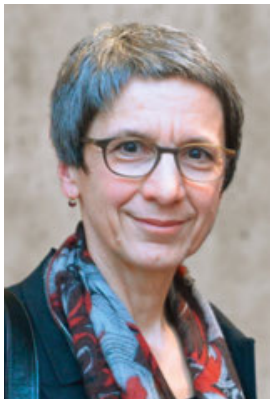

La saison des changements de caisse-maladie est de retour. L'occasion, en général, d'explorer les raisons et les responsables de l'augmentation des coûts de santé et les mesures urgentes à prendre pour maîtriser cette situation déplorable. Mais je promets que désormais, je renonce à accuser et à m'exprimer sur les erreurs de décision concernant la politique de la santé. Vous vous demandez peut-être ce qui étouffe ma tendance à m'imposer et me pousse à m'éloigner de l'agitation des expertes et des experts.

Le rapport d'experts «Mesures visant à freiner la hausse des coûts dans l'assurance obligatoire des soins», publié fin octobre par le Département fédéral de l'intérieur DFI, a motivé ma réticence. Ou plutôt, les réactions qu'il a suscitées. A peine était-il paru que les représentants d'intérêts se sont manifestés. Les médecins, patients, caisses-maladie, hôpitaux et pharmacies, ainsi que l'industrie pharmaceutique, ont aussitôt dégainé pour s'élever à l'unisson contre la menace de "rationnement» et de «médecine à deux vitesses». "Ce serait l'abandon d'un système de santé solidaire en Suisse», écrivaient les signataires du communiqué de presse, parmi lesquels ceux connus jusque-là pour prôner plus de concurrence et de responsabilité individuelle. La cible principale de ces attaques concertées est la proposition du rapport de fixer un objectif global d'évolution des coûts, en un mot un budget global. L'idée est de ralentir progressivement la croissance des coûts de l'assurance de base obligatoire en limitant les quantités, de façon à ce qu'à terme les primes des caisses-maladie continuent à augmenter au même rythme que les salaires.

\section{A peine était-il paru que les représentants d'intérêts se sont manifestés.}

Je me suis dans un premier temps étonnée de ce sursaut d'indignation de l'establishment de la santé. J'ai ensuite étudié le rapport. Un groupe d'experts internationaux y a rédigé un ensemble de 38 mesures concrètes, des nouvelles ou d'autres déjà en discussion depuis longtemps. Elles sont classées par ordre de priorité, clairement décrites et argumentées, avec de nombreux exemples étrangers. J'ai par exemple été frappée par le renoncement aux bonus et kickbacks liés aux quantités qui conditionnent l'inscription sur les listes hospitalières cantonales. L'idée d'introduire un «article expérimental» dans la LAMal est elle aussi intéressante, car elle permettrait par exemple de tester de nouveaux modèles de rétribution. Les autrices et auteurs abordent également la question des comités médicaux (Indikationsboards), un instrument incontesté sur le principe, mais rarement utilisé à ce jour (sauf en oncologie), car il touche des intérêts économiques.

\section{En regardant de plus près, on découvre aussi un grand souci de transparence et de qualité.}

Certes, les 38 propositions ne sont pas toutes pareillement convaincantes. Mais dans l'ensemble, ce train de mesures est soigneusement ficelé. Le titre du rapport indique en effet que l'objectif est de réduire les coûts. En regardant de plus près, on découvre aussi un grand souci de transparence et de qualité. Maintenant, si les principaux représentants d'intérêts se concentrent sur une mesure centrale, dans le meilleur des cas, c'est parce qu'ils n'ont pas encore trouvé le temps de lire le rapport en entier. Au pire, je dois en conclure que les profiteurs d'un système non transparent, inefficace et replié sur lui-même font tout ce qu'ils peuvent pour préserver leurs acquis.

Tout est dit pour ce qui est de l'évolution des coûts de la santé et de ses explications. Pour l'instant, je n'ai rien à ajouter. Donc cela tombe bien que mon mandat de rédactrice au Bulletin des médecins suisses se termine et que ces lignes constituent la dernière rubrique «Et encore...» que je rédige. Après dix années à vos côtés, je prends congé, chères lectrices et chers lecteurs. Je vous remercie chaleureusement pour tous vos commentaires critiques, dynamisants et constructifs. 